\title{
A Product Optimization Design Method to Respond Rapidly to Customer Requirements
}

\author{
Li Rupeng ${ }^{1}$, Wei Wei ${ }^{2}$, Zhou Feng $^{2}$, Zou Cheng ${ }^{1}$ \\ 1. Shanghai Aircraft Manufacturing Co., Ltd., Shanghai 200436, China. \\ 2. School of Mechanical Engineering and Automation, Beihang University, Beijing 100191, China.
}

\begin{abstract}
In the current highly competitive market environment, a critical success factor for enterprises is the ability to respond rapidly to customer requirements (CRs). This paper proposes a novel method to rapidly respond to CRs in product optimization design using fuzzy clustering and conjoint analysis-quality function deployment (CA-QFD). The approach we propose has two key characteristics. The first is classifying original complex CR data as a standard CR dataset with the fuzzy clustering method. The second is a new CA-QFD transformation method that integrates conjoint analysis with traditional QFD and can accurately transform CRs into product design attributes. Finally, to demonstrate the validity of the proposed method, we conduct a product optimization experiment by forging a machine's main hydraulic cylinder.
\end{abstract}

Keywords: rapid response to customer requirements; fuzzy clustering; requirements transformation; CA-QFD method; product optimization design

\section{Introduction}

Customer requirements (CRs) are the starting point and driving force for personalized customization on a large scale. Product optimization design based on CRs is the inevitable choice for modern enterprises to adapt to market development. However, with the rapid development of the market, CRs have become more diverse, personalized, and fuzzy, and it has become increasingly difficult to quantify them. This makes it difficult for companies to respond quickly and effectively to CRs when designing products. Therefore, the effective conversion of CR attributes into product design attributes is extremely important if a company's product optimization design is to respond quickly to $\mathrm{CRs}$.

In the last few years, scholars have had differing opinions on the issues of requirements transformation and dynamic requirements uncertainty. Luo et al. [1] propose a product design approach based on QFD for determining the optimal target levels of engineering characteristics with reference to CRs. Sheng et al. [2] propose a method based on the House of Quality for mapping from CRs to technical weights and target values. Violante et al. [3] propose an approach to obtain company-specific demands and determine the right features by integrating QFD with the Kano model. He et al. [4] improve the traditional Kano model and break through the limitations of dealing with the ambiguity and uncertainty of human thinking in a multigranularity language environment in the field of understanding customer needs. Wang and Tseng [5] propose a naive Bayes classifier-based approach, using existing customer choices data, for mapping CRs to specific product design variants. In order to better transform CRs into technical characteristics, Zaim et al. [6] propose a hybrid method that integrates analytic network process (ANP)-weighted QFD with fuzzy logic to better rank the technical characteristics of products. Li et al. [7] propose an

Received date: February 13, 2018; Revised date: March 30, 2018

Corresponding author: Wei Wei, School of Mechanical Engineering and Automation, Beihang University, Lecturer. Major research fields include product family design, and digital design and manufacturing. E-mail: weiwei@buaa.edu.cn

Funding program: CAE Advisory Project “Research on Development Strategy of 'Internet Plus' Action Plan”(2016-ZD-03); Project of National Natural Science Foundation of China (51675028); Project of National Key R\&D Plan (2017YFB1104200)

Chinese version: Strategic Study of CAE 2018, 20 (2): 033-041

Cited item: Li Rupeng et al. A Product Optimization Design Method to Respond Rapidly to Customer Requirements. Strategic Study of CAE, https://doi.org/10.15302/ J-SSCAE-2018.02.006 
approach unifying rough set methodology with QFD for modeling relationship measures in the process of product planning. Many variants of CR modeling methodologies have been proposed, such as QFD [8], probability analysis [9,10], and cluster analysis [11].

In previous research, customer demand was original information obtained directly from the client. However, original customer demand data is often ambiguous and overlapping, and cannot be used directly. The process of transforming CRs into product design attributes is independent for each reference condition. However, in real life, the final decision of a customer on a product is the result of comprehensive considerations and trade-offs for each reference condition. Targeting these problems, this paper proposes a method for rapid response to customer demands based on fuzzy clustering and CA-QFD. First, we cluster the original customer demand information by fuzzy clustering, and filter the results by enterprise to obtain the customer demand set. Then, through the joint analysis phase of the constructed demand transformation method, we quantify the customer's demand, weight it, and then transform it into a product design attribute through the QFD conversion stage. Finally, we optimize the product using a multi-objective optimization method to achieve rapid responses to CRs.

\section{Fuzzy clustering of customer requirements}

\subsection{Fuzzy clustering model of CRs}

Clustering classifies a target group according to the degree of similarity. The objects in the same class have a high degree of similarity, and the objects in different classes have large differences. In this paper, we use the fuzzy clustering method based on fuzzy similarity theory to cluster the original customer demand information. Fuzzy clustering obtains customer demand information as a starting point. Through the standardization of demand information, the optimal clustering scheme is obtained. The model is shown in Fig. 1.

\subsubsection{Obtaining CR information}

The principles of acquiring customer demand information is as follows: (i) The coverage principle: for all objects listed as customers, to provide full coverage of all requirements. (ii) The suggestion principle: in the process of obtaining the demand information, to help customers clearly express their needs by using suggestive language. (iii) The refinement principle: customers' requirements for each attribute of the product are specifically refined.
The method to acquire customer demand information is as follows: (i) External market surveys: customer surveys, telephone surveys, and other methods to discover customer needs. (ii) Enterprise database information analysis: analysis and forecasting of enterprise historical orders and customer feedback data (iii) Internet mode acquisition: access to customer needs through the Internet platforms.

\subsubsection{CR information standardization}

The original demand information obtained directly from the client is often unclear, and demand attributes overlap and cross. Standardization converts the customer's demand information into usable information.

\subsubsection{CR fuzzy clustering}

The fuzzy clustering method clusters various CRs, transforming the customer's needs from a single object into a whole composed of multiple objects with high degrees of similarity.

\subsubsection{Clustering result selection}

An expert evaluation of the enterprise selects the best solution from the dynamic clustering results as the customer demand set.

\subsection{CR clustering based on fuzzy similarity theory}

Assume that $n \mathrm{CR}$ attributes to be clustered constitute a set $C=\left\{a_{1}, a_{2}, \ldots, a_{n}\right\}$, where $a_{i}$ represents a $\mathrm{CR}$ attribute and $C$ is a finite non-empty set. Assume that $m$ customer objects to be clustered constitute a set $X=\left\{x_{1}, x_{2}, \ldots, x_{m}\right\}$, where $x_{i}$ represents a customer object and $X$ is a finite non-empty set. Then $S=(X, C)$ is defined as the CR information system.

\subsubsection{Standardizing requirement information}

The original customer demand information is divided into two types: ladder and equal. Ladder type information refers to the hierarchical relationship of demand attribute values, such as weight and efficiency. Equal type information means that the attribute values are independent of each other, and there is no difference in their relationship level, such as the customer's choice of product packaging color.

The ladder-type attributes are gathered in the front of the information system, the equal-type attributes are gathered in the back, and they are processed using standardized mathematical models. A mathematical model for information standardization is established as follows:

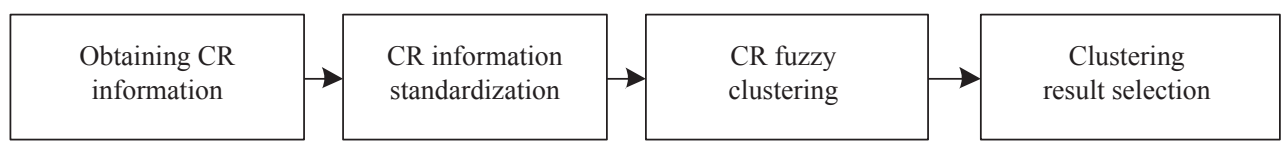

Fig. 1. Fuzzy clustering process of CRs. 


$$
x_{i k}= \begin{cases}\frac{x_{i k}^{\prime}-x_{k \min }^{\prime}}{x_{k \max }^{\prime}-x_{k \min }^{\prime}}, k=1,2, \cdots, l \\ x_{i k}^{\prime}, \quad k=l+1, l+2, \cdots n\end{cases}
$$

where $i=1,2, \ldots, m, x^{\prime}$ represents original information data, and $x$ represents standardized information data.

\subsubsection{Establishing the fuzzy similar matrix}

Attribute values that are of equal type are converted using the mean of the ladder type attribute set at the front of the information system:

$$
b_{k}=\frac{1}{\operatorname{lm}\left(h_{k}-1\right)} \sum_{i=1}^{m} \sum_{k=1}^{l} \frac{x_{i k}^{\prime}-x_{k \text { min }}^{\prime}}{x_{k \text { max }}^{\prime}-x_{k \text { min }}^{\prime}}
$$

where $k=l+1, l+2, \ldots, n$ and $h_{k}$ represents the $k$ th requirement attribute value type.

$$
q_{i j}^{(k)}= \begin{cases}0, & a_{k} \text { is same } \\ b_{k}, & a_{k} \text { is different }\end{cases}
$$

where $a_{k}$ represents the equality type requirement attribute value and $k=l+1, l+2, \ldots, n$.

In the customer set $X=\left\{x_{1}, x_{2}, \ldots, x_{m}\right\}$ the fuzzy similarity value $r_{i j}$ between customer $x_{i}$ and $x_{j}$ is obtained below:

$$
r_{i j}= \begin{cases}1, & i=j \\ 1-c\left(\sum_{k=1}^{l}\left|x_{i k}-x_{j k}\right|+\sum_{l+1}^{n} q_{i j}^{(k)}\right), & i \neq j\end{cases}
$$

where $c$ represents correction factor $0 \leqslant r_{i j} \leqslant 1$.

The fuzzy similar matrix is obtained below:

$$
\boldsymbol{R}=\left[\begin{array}{cccc}
r_{11} & r_{12} & \cdots & r_{1 m} \\
r_{21} & r_{22} & \cdots & r_{2 m} \\
\vdots & \vdots & \ddots & \vdots \\
r_{m 1} & r_{m 2} & \cdots & r_{m m}
\end{array}\right]
$$

The matrix has the following characteristics: reflexivity $\forall i=$ $j, r_{i j}=1$, symmetry $r_{i j}=r_{j i}$, and both equal the fuzzy similarity value between $x_{i}$ and $r_{j}$.

\subsubsection{Establishing the closure matrix}

The fuzzy similarity matrix is transitive, the closure matrix $t(\boldsymbol{R})$ of $\boldsymbol{R}$ is the fuzzy equivalent matrix. That is, it satisfies the condition of $r_{i j} \wedge r_{j k} \leqslant r_{i k},\left(\boldsymbol{R} \times \boldsymbol{R} \subseteq \boldsymbol{R}^{2}\right)$. The method of obtaining the fuzzy equivalent matrix is to square $\boldsymbol{R}$ to obtain $\boldsymbol{R} \times \boldsymbol{R}=\boldsymbol{R}^{2}$, and square $\boldsymbol{R}^{2}$, until it appears $\boldsymbol{R}^{k} \circ \boldsymbol{R}^{k}=\boldsymbol{R}^{k}$.

$$
t(\boldsymbol{R})=\boldsymbol{R}^{k}
$$

\subsubsection{Cut-set matrix}

Cut-set matrix $\boldsymbol{R}_{\lambda}=\left(\lambda_{i j}\right)$

$$
\lambda_{i j}= \begin{cases}1, & r_{i j} \geqslant \lambda \\ 0, & r_{i j}<\lambda\end{cases}
$$

where $i, j=1,2, \ldots, n$, the value set of $\lambda$ is the value of the element in the closure matrix $t(\boldsymbol{R})$, and we obtain dynamic clustering results through the different values of $\lambda$. The experts' evaluation selects the optimal clustering result, and obtains the CR set $F_{C R}=\left(C R_{1}, C R_{2}, \ldots, C R_{m}\right)$, where $m$ represents the clustering number of the optimal clustering result.

\section{CR transformation based on CA-QFD}

\subsection{The description of CA-QFD}

QFD is the classic method to transform CRs into product design attributes. However, in the traditional QFD method, customers evaluate each product's needs independently in the customer survey. Although this is beneficial and reduces the complexity of the requirement quantification problem, the result is only the customer's partial preference for each attribute, rather than their overall preference for the entire product. The reason is that, when customers make decisions on products, they are the result of a comprehensive consideration of multiple demand situations of the products, which involves a trade-off of requirements. Evaluating attributes completely independently adversely affects the applicability and authenticity of the final quantitative results of the requirements.

Conjoint analysis uses the product model attribute considered in the customer's decision-making as a factor at the customer survey stage, and takes the customer's preference for the factor as a utility. By providing customers with product portfolios that include different attributes for comparison and evaluation, their preferences for different product attributes are obtained. Customers can comprehensively consider multiple features in product decision making and make up for the shortcomings of traditional QFD methods in requirement survey analysis.

This paper combines conjoint analysis and QFD to construct a new requirement transformation method, CA-QFD. The method is outlined below:

At the conjoint analysis stage:

Step 1: Determine factors and the levels of factors

Step 2: Determine the product profile and obtain the customer's evaluation results

Step 3: Mathematically model the customer's requirement weights

At the QFD stage:

Step 4: Quantify the relationship between the factors and the design attributes

Step 5: Calculate the design attribute weights and construct a QFD transformation matrix

\subsection{CR attribute quantization at the conjoint analysis stage}

The conjoint analysis first determines the reference factors when the customer selects the product, and determines the ap- 
propriate factor level for each factor. This paper takes the standard customer demand obtained by fuzzy clustering as a factor, and matches each factor with no more than four levels. The orthogonal design method of statistical package for the social sciences (SPSS) tools in conjoint analysis generates a representative product profile that improves the accuracy of the survey response and data. At the same time, using the scoring method, it produces a $0-100$ interval ruler table where 0 means most dissatisfied and 100 means most satisfied.

We analyze and calculate the scores of the product profiles through the conjoint analysis method, and obtain the utility of the factor level. The mathematical model is as follows:

$$
U_{k}(X)=\sum_{i=1}^{I} \sum_{l=1}^{L_{i}} P W_{k i l} X_{i l}
$$

where $U_{k}(X)$ represents the total utility of a product profile; $I$ represents the total number of factors; $P W_{k i l}$ represents the utility of the level $l$ of factor $i$ in customer group $k, L_{i}$ represents the total number of levels of factor $i$, and $X_{i l}$ represents the determined value.

$$
X_{i l}= \begin{cases}1, & \text { level of } i \text { exists } \\ 0, & \text { level of } i \text { does not exist }\end{cases}
$$

Each factor has a utility value at its corresponding level. Then, we quantify the importance of CR attributes through the conjoint analysis: if there is no difference between all the utility values of factor $i$, it means that factor $i$ has little influence on the customer's choice of products. Conversely, if there is a great difference between each utility value, it means that factor $i$ has a great influence on the customers' choice of products.

Calculate the CR weight as follows:

$$
w_{k i}^{C R}=\frac{\max P W_{k i l}-\min P W_{k i l}}{\sum_{i=1}^{I}\left(\max P W_{k i l}-\min P W_{k i l}\right)}
$$

where $w_{k i}^{C R}$ represents the CR weight of customer $i$ in the $k$ th customer group, and $\max P W_{k i l}$ and $\min P W_{k i l}$ represent the maximum and minimum factor level value, respectively.

\subsection{The QFD Matrix for transforming CR attributes}

We quantify the correlation degree between CR attributes and design attributes and the coupling degree between each design attribute by the scale table 0-1-3-5-7-9. Then, we complete the transformation by importing the quantification results into the QFD transformation matrix.

To obtain the QFD matrix, first quantify the incidence re- lation between $\mathrm{CR}$ attributes $\left(C R_{i}\right)$ and design attributes $\left(f_{i}\right)$ through the scale table $0-1-3-5-7-9$. The related intensity can be divided into six levels, and the values can be used to define the intensity, as in Table 1.

The mathematical model of the design attribute weighting is below:

$$
w_{k j}^{D A}=\frac{\sum_{i=1}^{m} w_{k i}^{C R} r_{i j}}{\sum_{j=1}^{n} \sum_{i=1}^{m} w_{k i}^{C R} r_{i j}}
$$

where $w_{k j}^{D A}$ represents the weight of design attribute $j$ in the $k$ th customer group and $r_{i j}$ represents the value of the related intensity.

The QFD matrix of design attribute weights is given below:

\begin{tabular}{ccccccc}
\hline \multirow{2}{*}{$C R$} & \multirow{2}{*}{$w_{k i}^{C R}$} & \multicolumn{5}{c}{ Design Attributes } \\
\cline { 3 - 7 } & & $f_{1}$ & $\cdots$ & $f_{j}$ & $\cdots$ & $f_{n}$ \\
\hline$C R_{1}$ & $w_{k 1}^{C R}$ & $r_{11}$ & $\cdots$ & $r_{1 j}$ & $\cdots$ & $r_{1 n}$ \\
$\cdots$ & $\cdots$ & $\cdots$ & & $\cdots$ & & $\cdots$ \\
$C R_{i}$ & $w_{k i}^{C R}$ & $r_{1 i}$ & $\cdots$ & $r_{i j}$ & $\ldots$ & $r_{i n}$ \\
$\ldots$ & $\ldots$ & $\cdots$ & & $\cdots$ & & $\cdots$ \\
$C R_{m}$ & $w_{k m}^{C R}$ & $r_{1 m}$ & $\cdots$ & $r_{m j}$ & $\cdots$ & $r_{m n}$ \\
\hline \multicolumn{2}{l}{$\begin{array}{c}\text { Design attribute } \\
\text { weights }\end{array}$} & $w_{k 1}^{D A}$ & $\cdots$ & $w_{k j}^{D A}$ & $\cdots$ & $w_{k n}^{D A}$ \\
\hline
\end{tabular}

The design attribute set is $F=\left(f_{1}, f_{2}, \ldots, f_{n}\right)$, and the enterprise selects the design attributes according to importance factor $b$. If the weight value of the design attribute is greater than $b$, then it means that the attribute is sufficiently important to require a response, so it will be chosen as the optimization objective.

\section{The multi-objective product optimization design model}

Product optimization design for CRs is an extension of the multi-objective optimization problem. The mathematical model is given below:

$$
\begin{array}{lrl}
\min F(x) & =\left(f_{1}(x), f_{2}(x), \cdots, f_{r}(x)\right) \\
\text { s.t. } & h(x)=0, \\
& g(x)=0, \\
& x^{\min } \leqslant x \leqslant x^{\max }
\end{array}
$$

This paper uses the NSGA-II [12,13] algorithm to independently optimize each product to obtain the Pareto optimal set after CRs are transformed into product optimization objectives,

Table 1. Related intensity and the corresponding value evaluation index.

\begin{tabular}{lcccccc}
\hline Intensity & Very strong & Moderately strong & Strong & Weak & Very weak & Independent \\
\hline Value & 9 & 7 & 5 & 3 & 1 \\
\hline
\end{tabular}


and then to obtain an optimal solution according to fuzzy set theory. The NSGA-II genetic algorithm is shown in Fig. 2.

The algorithm has the characteristics of strong stability and fast computing speed. It is used in engineering optimization design, and is an effective solution to optimization design problems with multiple objectives. It first sorts the initial population and calculates the crowded distance, then generates new individuals through genetic operations such as crossover and mutation. The new individual and the parental band are fused to form a new generation of temporary storage population, then the temporary population is sorted according to rank and crowding distance and an evolutionary operation is completed. When the loop reaches the preset number of iterations, the operation stops and obtains the optimal solution set of the optimization target. Finally, the optimal solution is filtered by the fuzzy set.

\section{Case illustration}

\subsection{Fuzzy clustering of hydraulic cylinder CRs}

According to the market requirement statistics for the main hydraulic cylinder of forging machines, the original requirement information statistic table of the 12 forging-machine manufacturing enterprises is obtained, as shown in Table 2.

$a_{1}$ represents price, $a_{2}$ represents allowable stress of hydraulic cylinder, $a_{3}$ represents weight, $a_{4}$ represents minimum service life, $a_{5}$ represents maximum stroke, and $a_{6}$ represents the hydraulic cylinder usage attribute value, where 1,2 , and 3 represent the normal, medium, and large special types, respectively.

Let $c=0.1$, and obtain the closure matrix $t(\boldsymbol{R}), t(\boldsymbol{R})=\boldsymbol{R}^{8}$ from equations (1) through (6).

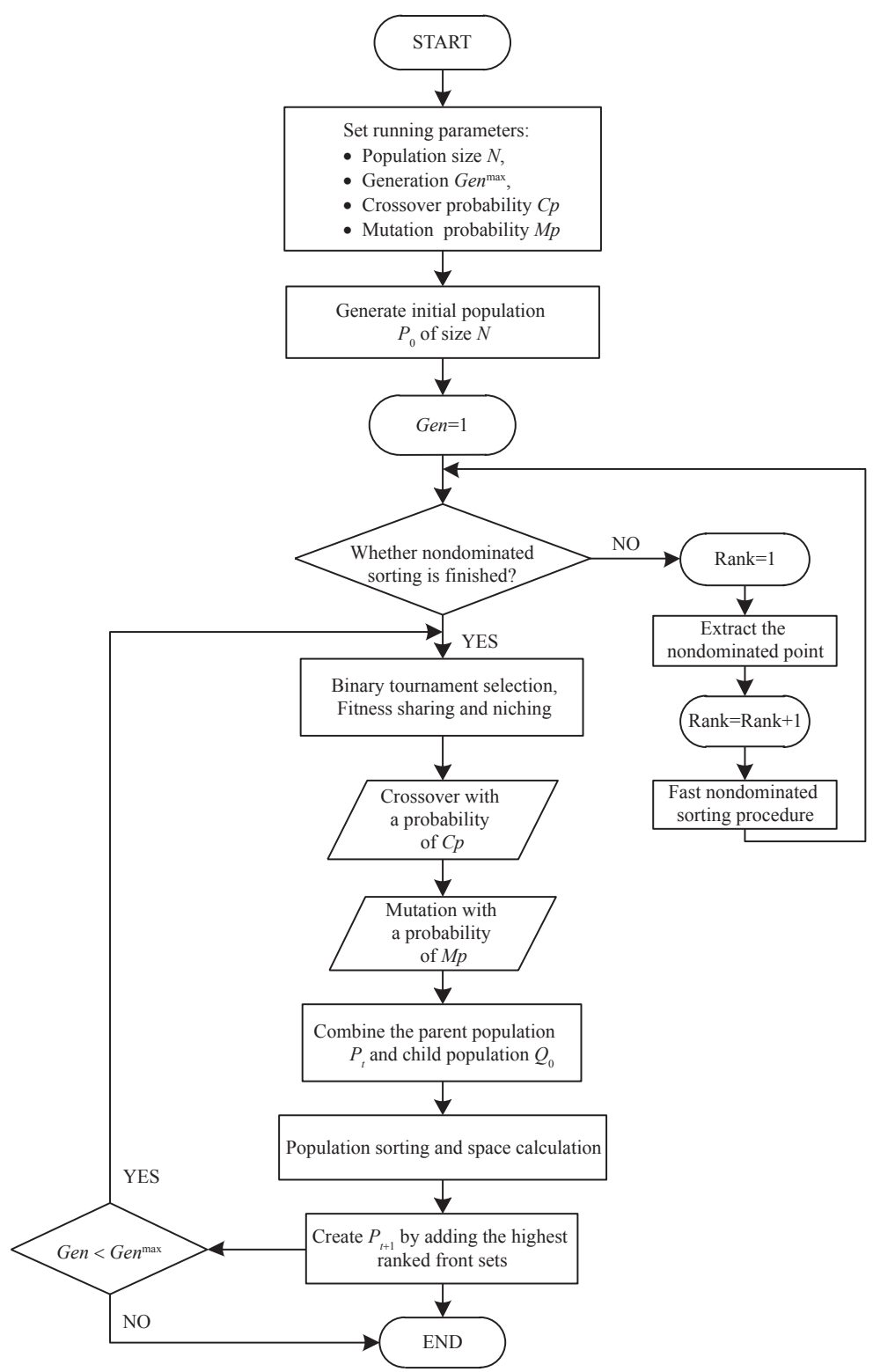

Fig. 2. The NSGA-II process. 
Table 2. Original requirement information statistics.

\begin{tabular}{|c|c|c|c|c|c|c|}
\hline Customer & $a_{1}(10000$ yuan $)$ & $a_{2}(\mathrm{Mpa})$ & $a_{3}(\mathrm{t})$ & $a_{4}$ (year) & $a_{5}(\mathrm{~m})$ & $a_{6}$ \\
\hline $\mathrm{x}_{1}$ & 6 & 120 & 55 & 5.5 & 1.5 & 1 \\
\hline $\mathrm{x}_{2}$ & 22 & 100 & 49 & 11 & 1.5 & 2 \\
\hline $\mathrm{x}_{3}$ & 7 & 120 & 53 & 7 & 1.25 & 1 \\
\hline $\mathrm{x}_{4}$ & 9 & 115 & 55 & 6 & 1.5 & 1 \\
\hline $\mathrm{x}_{5}$ & 2.5 & 135 & 60 & 3 & 1 & 1 \\
\hline $\mathrm{x}_{6}$ & 16 & 105 & 51 & 9 & 1 & 2 \\
\hline $\mathrm{x}_{7}$ & 15 & 100 & 50 & 10 & 1.75 & 2 \\
\hline $\mathrm{x}_{8}$ & 35 & 80 & 40 & 14 & 2 & 3 \\
\hline $\mathrm{x}_{9}$ & 2 & 130 & 62 & 4 & 1.25 & 1 \\
\hline $\mathrm{x}_{10}$ & 8 & 120 & 54 & 6 & 1.5 & 1 \\
\hline $\mathrm{x}_{11}$ & 7.5 & 115 & 53 & 5 & 2 & 1 \\
\hline $\mathrm{x}_{12}$ & 10 & 110 & 50 & 8 & 1.5 & 2 \\
\hline
\end{tabular}

$t(\boldsymbol{R})=\left[\begin{array}{llllllllllll}1.00 & 0.92 & 0.95 & 0.93 & 0.95 & 0.92 & 0.92 & 0.85 & 0.95 & 0.98 & 0.95 & 0.95 \\ 0.92 & 1.00 & 0.92 & 0.92 & 0.92 & 0.93 & 0.93 & 0.85 & 0.92 & 0.92 & 0.93 & 0.92 \\ 0.95 & 0.92 & 1.00 & 0.93 & 0.95 & 0.92 & 0.92 & 0.85 & 0.95 & 0.95 & 0.93 & 0.92 \\ 0.93 & 0.92 & 0.93 & 1.00 & 0.93 & 0.92 & 0.92 & 0.85 & 0.93 & 0.96 & 0.95 & 0.98 \\ 0.95 & 0.92 & 0.95 & 0.93 & 1.00 & 0.92 & 0.92 & 0.85 & 0.92 & 0.93 & 0.93 & 0.93 \\ 0.92 & 0.93 & 0.92 & 0.92 & 0.92 & 1.00 & 0.98 & 0.85 & 0.92 & 0.96 & 0.95 & 0.95 \\ 0.92 & 0.93 & 0.92 & 0.92 & 0.92 & 0.98 & 1.00 & 0.85 & 0.85 & 0.92 & 0.92 & 0.92 \\ 0.85 & 0.85 & 0.85 & 0.85 & 0.85 & 0.85 & 0.85 & 1.00 & 0.85 & 0.92 & 0.92 & 0.92 \\ 0.95 & 0.92 & 0.95 & 0.93 & 0.92 & 0.92 & 0.85 & 0.85 & 1.00 & 0.85 & 0.85 & 0.95 \\ 0.98 & 0.92 & 0.95 & 0.96 & 0.93 & 0.96 & 0.92 & 0.92 & 0.85 & 1.00 & 0.85 & 0.95 \\ 0.95 & 0.93 & 0.93 & 0.95 & 0.93 & 0.95 & 0.92 & 0.92 & 0.85 & 0.85 & 1.00 & 0.95 \\ 0.95 & 0.92 & 0.92 & 0.98 & 0.93 & 0.95 & 0.92 & 0.92 & 0.95 & 0.95 & 0.95 & 1.00\end{array}\right]$

According to matrix $t(\boldsymbol{R})$, calculate the set of $\lambda:\{1.00,0.98$, $0.96,0.95,0.93,0.92,0.85\}$. The results from the dynamic clustering of the CRs are shown in Fig. 3.

According to the evaluation results, when $\lambda=0.93, r_{\lambda}=4$, the demand importance index $F_{\lambda}$ is best. Therefore, the optimal clustering result is $\left\{\mathrm{x}_{1}, \mathrm{x}_{3}, \mathrm{x}_{4}, \mathrm{x}_{10}, \mathrm{x}_{11}\right\},\left\{\mathrm{x}_{2}, \mathrm{x}_{6}, \mathrm{x}_{7}, \mathrm{x}_{12}\right\},\left\{\mathrm{x}_{5}, \mathrm{x}_{9}\right\}$, $\left\{\mathrm{x}_{8}\right\}$. If each cluster subset is defined as a CR factor, then the best set of CRs is obtained from $F_{C R}=\left(C R_{1}, C R_{2}, C R_{3}, C R_{4}\right)$, where $C R_{1}$ represents price factor, $C R_{2}$ represents safety factor, $C R_{3}$ represents weight factor, and $C R_{4}$ represents lifetime factor.

\subsection{CR transformation}

Take the CR set obtained by fuzzy clustering above as the factor of conjoint analysis, and then determine factor levels, as shown in Table 3 for the hydraulic cylinder market.

Use the conjoint analysis method to establish a total combination profile of factor levels and market samples score for each profile, sample size 50. The evaluation results of the profile are shown in Table 4.

Using formulas (8) through (10), we obtain the utility value of each factor level and the weight of CRs. The results are shown in Table 5.
The main hydraulic cylinder's design attributes and forging machine metrics are mainly the following: $f_{1}$ is power loss $\Delta P$ (economic attribute index); $f_{2}$ is flange transition zone maximum equivalent stress $Y D / \mathrm{MPa}$ (safety attribute index); $f_{3}$ is friction torque $M f / \mathrm{N} \cdot \mathrm{cm}, f_{4}$ is fatigue stress $\sigma / \mathrm{MPa}$ (lifetime attribute index); $f_{5}$ is volume $V / \mathrm{cm}^{3}$ (weight attribute index).

Assign the CR attributes to design attributes by using the quantitative scale 0-1-3-5-7-9, and obtain the design attribute weights from formula (11). The QFD matrix is shown in Fig. 4.

For hydraulic cylinder manufacturing enterprises, the importance factor value $b$ is 0.2 . Design attributes that are bigger than $b$ should be selected as the optimization objective. Therefore, the optimization objectives in this case are $f_{2}$ and $f_{5}$.

\subsection{Multi-objective optimization design of hydraulic cylinder}

Through the previous steps of clustering and quantifying the design attributes, we find the optimization objectives to be safety and weight. Next, we replace safety with the maximum equivalent stress in the flange transition zone $Y D$, and weight with the hydraulic cylinder volume $V$. This establishes the optimization objective function and constraints for the hydraulic cylinder. A simplified model of the hydraulic cylinder is shown in Fig. 5. 


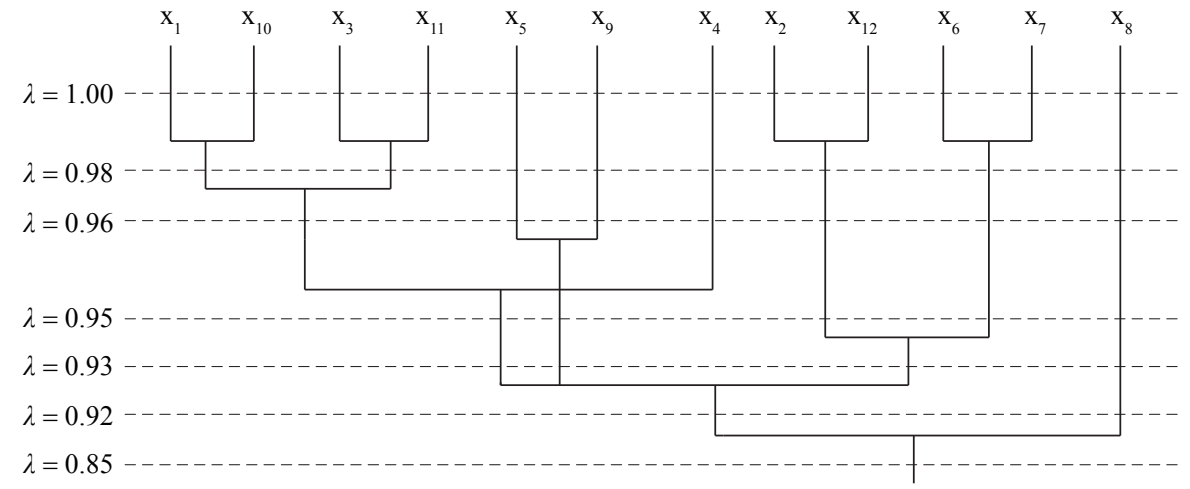

Fig. 3. The dynamic clustering results.

Table 3. Factor and factor levels.

\begin{tabular}{llll}
\hline & Factor & & \multicolumn{1}{c}{ Factor Level } \\
\hline$C R_{1}$ & Price & $\mathrm{L}_{11}$ & High \\
& & $\mathrm{L}_{12}$ & Middle \\
& & $\mathrm{L}_{13}$ & Low \\
$C R_{2}$ & Safety & $\mathrm{L}_{21}$ & Very important \\
& & $\mathrm{L}_{22}$ & Important \\
$C R_{3}$ & Weight & $\mathrm{L}_{31}$ & Heavy \\
& & $\mathrm{L}_{32}$ & Light \\
$C R_{4}$ & Lifetime & $\mathrm{L}_{41}$ & Long \\
& & $\mathrm{L}_{42}$ & Short \\
\hline
\end{tabular}

Table 4. The evaluation results of the profile.

\begin{tabular}{|c|c|c|c|c|c|c|c|c|c|c|c|c|c|c|c|c|c|c|c|}
\hline \multirow{2}{*}{$\mathrm{N} / \mathrm{S}$} & \multicolumn{3}{|c|}{$C R_{1}$} & \multicolumn{2}{|c|}{$C R_{2}$} & \multicolumn{2}{|c|}{$\mathrm{CR}_{3}$} & \multicolumn{2}{|c|}{$C R_{4}$} & \multicolumn{10}{|c|}{ Score } \\
\hline & $\mathrm{L}_{11}$ & $\mathrm{~L}_{12}$ & $\mathrm{~L}_{13}$ & $\mathrm{~L}_{21}$ & $\mathrm{~L}_{22}$ & $\mathrm{~L}_{31}$ & $\mathrm{~L}_{32}$ & $\mathrm{~L}_{41}$ & $\mathrm{~L}_{42}$ & 1 & 2 & 3 & 4 & 5 & 6 & 7 & 8 & 9 & $\ldots$ \\
\hline 1 & $\sqrt{ }$ & & & $\sqrt{ }$ & & $\sqrt{ }$ & & $\sqrt{ }$ & & 80 & 60 & 90 & 70 & 50 & 80 & 70 & 90 & 60 & $\ldots$ \\
\hline$\ldots$ & & & & & & & & & & & & & & & & & & & $\ldots$ \\
\hline 8 & & $\sqrt{ }$ & & $\sqrt{ }$ & & $\sqrt{ }$ & & $\sqrt{ }$ & & 70 & 50 & 90 & 50 & 60 & 70 & 80 & 90 & 90 & $\ldots$ \\
\hline$\ldots$ & & & & & & & & & & & & & & & & & & & $\ldots$ \\
\hline 16 & & $\sqrt{ }$ & & & $\sqrt{ }$ & & $\sqrt{ }$ & & $\sqrt{ }$ & 90 & 60 & 80 & 70 & 90 & 90 & 50 & 60 & 40 & $\ldots$ \\
\hline$\cdots$ & & & & & & & & & & & & & & & & & & & $\ldots$ \\
\hline 24 & & & $\sqrt{ }$ & & $\sqrt{ }$ & & $\sqrt{ }$ & & $\sqrt{ }$ & 50 & 90 & 60 & 90 & 60 & 80 & 60 & 50 & 70 & $\ldots$ \\
\hline
\end{tabular}

Table 5. Weights of CRs.

\begin{tabular}{lccc}
\hline CRs & Level & Utility & Weight \\
\hline$C R_{1}$ & $\mathrm{~L}_{11}$ & +0.160 & 0.200 \\
& $\mathrm{~L}_{12}$ & +0.052 & \\
& $\mathrm{~L}_{13}$ & -0.110 & \\
$C R_{2}$ & $\mathrm{~L}_{21}$ & +0.016 & 0.348 \\
& $\mathrm{~L}_{22}$ & +0.025 & \\
$C R_{3}$ & $\mathrm{~L}_{31}$ & +0.056 & 0.252 \\
& $\mathrm{~L}_{32}$ & +0.0127 & \\
$C R_{4}$ & $\mathrm{~L}_{41}$ & -0.052 & 0.200 \\
& $\mathrm{~L}_{42}$ & +0.104 & \\
\hline
\end{tabular}

The function of the optimization objective is illustrated as:

$$
\left\{\begin{aligned}
F(x)= & (\min Y D, \min V) \\
\min Y D= & \frac{6 P\left(R_{1}-R_{0}\right)}{\left(1+\frac{\beta H}{2}+\frac{1-\mu^{2}}{2 \beta R_{0}}\left(\frac{H}{t}\right)^{3} \ln \frac{R_{1}}{R_{0}}\right)\left(R_{0}+R_{2}\right) t^{2}} \\
& +\frac{P}{\pi\left(R_{2}^{2}-R_{0}^{2}\right)} \\
\min V= & \pi\left[H\left(R_{1}^{2}-R_{0}^{2}\right)+L\left(R_{2}^{2}-R_{0}^{2}\right)+T\left(R_{2}^{2}-R_{0}^{2}\right)\right] \\
+ & R_{0} \cdot R T^{2}+R_{2} \cdot R D^{2}
\end{aligned}\right.
$$

According to the design requirements, assembly process, pro- 


\begin{tabular}{c|ccccc|c}
\hline \multirow{2}{*}{ Customer requirement } & \multicolumn{5}{|c|}{ Design attributes } & Customer requirement weights $w_{k i}^{C R}$ \\
\cline { 2 - 5 } & $f_{1}$ & $f_{2}$ & $f_{3}$ & $f_{4}$ & $f_{5}$ & 0.200 \\
\hline$R_{1}$ & 5 & 9 & 3 & 5 & 7 & 0.348 \\
$C R_{2}$ & 7 & 5 & 0 & 0 & 1 & 0.252 \\
$C R_{3}$ & 1 & 5 & 9 & 3 & 3 & 0.200 \\
$C R_{4}$ & 1 & 3 & 0 & 3 & 9 & \\
\hline Design attribute weights $w_{k j}^{D A}$ & 0.176 & 0.241 & 0.165 & 0.173 & 0.245 \\
\hline
\end{tabular}

Fig. 4. The QFD matrix.

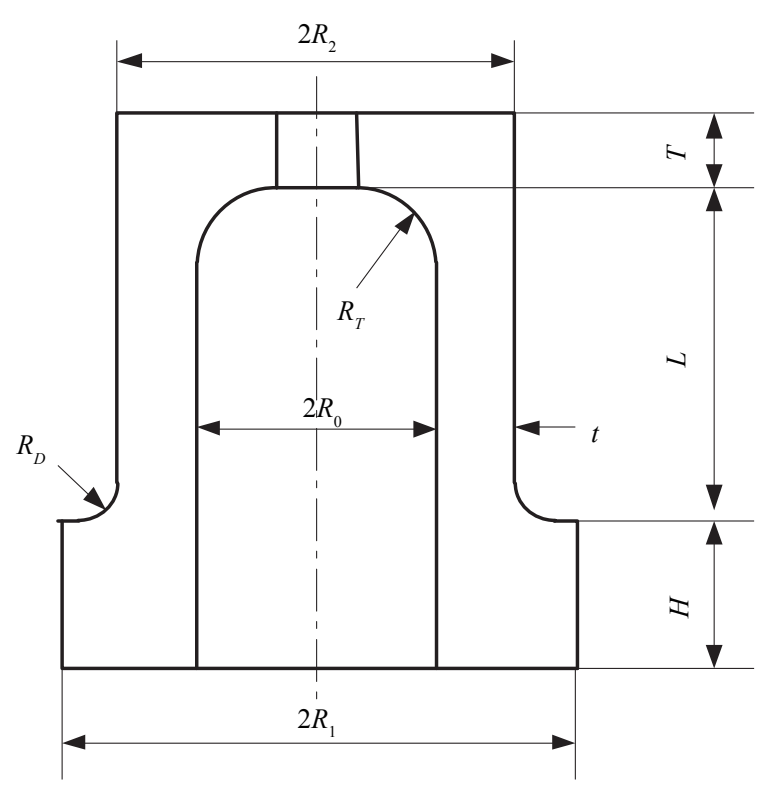

Fig. 5. A simplified model of the hydraulic cylinder.

cessing technology specification, and manufacturing experience of the main working hydraulic cylinder of the forging press, the inequality constraints of six design variables can be determined. The conditions of the design variables are shown in Table 6. In the table, $Y D$ represents the maximum equivalent stress in the flange transition zone, $Y G_{1}$ represents the allowable stress in the flange transition zone, $Y T$ represents the maximum calculated equivalent stress in the transition zone at the bottom of the cylin- der, $Y G_{2}$ represents the allowable stress in the bottom transition zone, $Y M$ represents the extrusion stress on the flange bearing surface, and $[Y M]$ represents allowable extrusion stress [14].

Based on the optimization model discussed above, NSGA-II is used to optimize every variable in the hydraulic cylinder. The population size of NSGA-II is 500, and the crossover and mutation probability are 0.8 and 0.5 , respectively. Their distribution indexes are both 20. The maximum iterations are 1000 .

All the experiments were run on a Pentium IV $(2.6 \mathrm{GHz})$ with 1 GB RAM using GNU/Linux, and took 30.9 s of CPU time. The result is presented and compared with the original product in Table 7.

As shown, the safety and weight performance of the hydraulic cylinder was optimized. The weight of the hydraulic cylinder was reduced by $22 \%$, and safety was increased by $3.8 \%$, which indicates that the proposed method can respond quickly to CRs.

\section{Conclusions}

This paper presents a new method to respond quickly to CRs for product optimization design, which starts with information about original CRs and ultimately obtains the optimization objective of the product.

First, the fuzzy clustering method proposed in this paper solves the problem of fuzzy and diverse CR classifications, and realizes the standardization and streamlining of the original CRs. This provides the conditions for the transformation of the

Table 6. Conditions of the design variable.

$\mathrm{cm}$

\begin{tabular}{lcc}
\hline Variable & Stress constraints & Range \\
\hline$R_{1}$ & $-1 \times 10^{20} \leqslant Y D-Y G_{1} \leqslant 0$ & $130 \leqslant R_{1} \leqslant 140$ \\
$H$ & $-1 \times 10^{20} \leqslant Y T-Y G_{2} \leqslant 0$ & $40 \leqslant H \leqslant 70$ \\
$T$ & $-1 \times 10^{20} \leqslant Y M-[Y M] \leqslant 0$ & $40 \leqslant T \leqslant 70$ \\
$R T$ & & $10 \leqslant R T \leqslant 40$ \\
$R D$ & & $0 \leqslant R D \leqslant 10$ \\
$t$ & & $20 \leqslant t \leqslant 50$ \\
\hline
\end{tabular}


Table 7. Optimization result.

\begin{tabular}{|c|c|c|c|c|c|c|c|c|c|c|c|c|c|c|c|}
\hline & \multicolumn{3}{|c|}{ Permissible stress (Mpa) } & \multicolumn{3}{|c|}{ Calculated stress (Mpa) } & \multicolumn{5}{|c|}{ Optimized size $(\mathrm{cm})$} & \multicolumn{2}{|c|}{ Bulk \&Weight } & \multicolumn{2}{|c|}{ Contrast } \\
\hline & {$[Y M]$} & $Y G_{I}$ & $Y G_{2}$ & $T M$ & $Y D$ & $Y T$ & $R_{1}$ & $H$ & $T$ & $R_{D}$ & $R_{T}$ & $\begin{array}{l}\text { Bulk } \\
\left(\mathrm{m}^{3}\right)\end{array}$ & $\begin{array}{l}\text { Weight } \\
(\mathrm{t})\end{array}$ & $\begin{array}{l}\text { Safety } \\
\text { (Mpa) }\end{array}$ & $\begin{array}{c}\text { Weight } \\
\text { (t) }\end{array}$ \\
\hline Original design & & & & 109 & 131 & 142 & 139 & 66 & 67 & 3.5 & 20 & 7.44 & 58.8 & & \\
\hline Optimized result & 100 & 160 & 40 & 100 & 126 & 140 & 134 & 69.1 & 40.2 & 1.5 & 26.2 & 5.88 & 45.9 & -5 & -12.9 \\
\hline
\end{tabular}

requirements. Second, the CA-QFD, the combination of the conjoint analysis and traditional QFD methods proposed in this paper, solves the traditional QFD method's insufficiency in independent analysis at the customer survey stage. CA-QFD also realizes the decision-making for the customer to select the product, which makes transformation results more accurate and more applicable. Finally, the example of the main working hydraulic cylinder of a forging press shows that the method proposed in this paper can quickly and accurately respond to CRs, obtain product optimization design schemes, and provide useful information for an enterprise's production.

\section{References}

[1] Luo X G, Kwong C K, Tang J F, et al. QFD-based product planning with consumer choice analysis [J]. IEEE Transactions on Systems Man \& Cybernetics Systems, 2015, 45(3): 454-461.

[2] Sheng Z, Wang Y, Song J, et al. Customer requirement modeling and mapping of numerical control machine $[\mathrm{J}]$. Advances in Mechanical Engineering, 2015, 7(10): 1-11.

[3] Violante M G, Vezzetti E, Alemanni M. An integrated approach to support the Requirement Management (RM) tool customization for a collaborative scenario $[\mathrm{J}]$. International Journal on Interactive Design \& Manufacturing, 2017, 11(2): 191-204.

[4] He L, Ming X, Li M, et al. Understanding customer requirements through quantitative analysis of an improved fuzzy Kano's model [J]. Journal of Engineering Manufacture, 2015, 231(4): 699-712.

[5] Wang Y, Tseng M M. A Naïve Bayes approach to map customer requirements to product variants [J]. Journal of Intelligent Manu- facturing, 2015, 26(3): 1-9.

[6] Zaim S, Sevkli M, Camgöz-Akdağ H, et al. Use of ANP weighted crisp and fuzzy QFD for product development [J]. Expert Systems with Applications, 2014, 41(9): 4464-4474.

[7] Li Y L, Tang J F, Chin K S, et al. Rough set-based approach for modeling relationship measures in product planning [J]. Information Sciences an International Journal, 2012, 193(11): 199-217.

[8] Chowdhury M M H, Quaddus M A. A multiple objective optimization based QFD approach for efficient resilient strategies to mitigate supply chain vulnerabilities: The case of garment industry of Bangladesh [J]. Omega, 2015 (57): 5-21.

[9] Wang Y, Tseng M M. Integrating comprehensive customer requirements into product design [J]. CIRP Annals- Manufacturing Technology, 2011, 60(1): 175-178.

[10] Wang Y, Tseng M M. Incorporating tolerances of customers' requirements for customized products [J]. CIRP Annals-Manufacturing Technology, 2014, 63(1): 129-132.

[11] Dong Y Y. Fuzzy cluster analysis [J]. Journal of Jinggangshan University, 2006, 62(10): 750-754.

[12] Wei W, Feng Y, Tan J, et al. Product platform two-stage quality optimization design based on multiobjective genetic algorithm [J]. Computers \& Mathematics with Applications, 2009, 57(11): 1929-1937.

[13 ] Chen Y L, Chu W L, Xu Y S. Adaptability-oriented parametric product platform design [J]. Computer Integrated Manufacturing Systems, 2007, 13(5): 877-884.

[14] Zhao S D, Wang J, Bai Z Y, et al. Optimum design of the structure of flange supported hydraulic cylinder by genetic algorithms $[\mathrm{J}]$. Forging \& Stamping Technology, 2008, 33(2): 95-99. Chinese. 